\title{
Migración de Fe y F en el acuífero de Apan, Hgo.
}

\section{U. García Padilla1 ${ }^{1}$ J.C. Escamilla Casas², E.M. Uribe Alcántara², S. Montiel Palma ${ }^{1 \star}$}

${ }^{1}$ Área Académica de Ingeniería y Arquitectura. Universidad Autónoma del Estado de Hidalgo, Carr. Pachuca-Tulancingo km. 4.5, Col. Carboneras, Mineral de la Reforma, Hidalgo, C.P. 42184, México. ga298235@uaeh.edu.mx,silvia_montiel@uaeh.edu.mx

${ }^{2}$ Área Académica de Ciencias de la Tierra y Materiales. Universidad Autónoma del Estado de Hidalgo, Carr. Pachuca-Tulancingo km. 4.5, Col. Carboneras, Mineral de la Reforma, Hidalgo, C.P. 42184, México. jocesca@uaeh.edu.mx,edgar_uribe8920@uaeh.edu.mx

*Autor de correspondencia: silvia_montiel@uaeh.edu.mx

\section{RESUMEN}

El acuífero Apan se localiza en la porción suroriental del Estado de Hidalgo. Debido a que han sido reportados valores de metales en pozos de abastecimiento público por encima de la norma mexicana para agua potable, este estudio se enfocó en analizar el comportamiento de estos posibles contaminantes en su recorrido por el acuífero.

Con información de los organismos locales, se analizaron los resultados para calcio, magnesio, sodio, potasio, cloruro, sulfato, carbonatos, bicarbonatos, fosfatos, aluminio, plomo, mercurio, cadmio, cobre zinc, hierro, manganeso boro, arsénico y fluoruros. De ellos únicamente los que se encontraban en cantidades por encima de los límites de detección del equipo fueron $\mathrm{Fe}, \mathrm{F}$ y en algunos casos $\mathrm{Mn}$ por lo que únicamente los dos primeros se consideraron en el estudio.

De acuerdo a dicha información, se corrobora que la facies hidrogeoquímica presente en la dirección del flujo subterráneo es del tipo bicarbonatada-mixta (bicarbonatada cálcica/magnésica a bicarbonatada sódica/cálcica), lo que indica un tipo de agua joven procedente del agua de lluvia y/o de flujo de tipo local con tendencia a un intercambio iónico entre $\mathrm{Ca}^{++} \mathrm{y} \mathrm{Mg}^{++}$.

El Fe presenta una tendencia de aumento en dirección espacial O-E. Los fluoruros presentan una tendencia ascendente con dirección concéntrica en el acuífero.

Palabras Clave: Acuífero, Contaminantes, Comportamiento, Hierro, Fluoruro.

\section{INTRODUCCIÓN}

El recurso hídrico, a nivel nacional, se encuentra altamente alterado tanto en su disponibilidad como en su calidad. Los factores principales que ocasionan su alteración son fundamentalmente la sobreexplotación de acuíferos, el vertido de sustancias contaminantes a los cuerpos de agua, los cambios en el uso del suelo tales como la deforestación, las prácticas agrícolas inadecuadas, así como también el incremento de urbanizaciones en zonas de recarga de acuíferos.

Por otra parte, las condiciones naturales, a través de la disolución de los minerales presentes en los medios geológicos por los que atraviesa el agua en su recorrido subterráneo; el cual puede llegar a ser de cientos o miles de años, puede llegar a 
ocasionar contaminación al rebasar ciertos límites a partir de los cuales tanto el consumo humano como el agrícola pudieran ser afectados.

En el municipio de Apan el agua subterránea representa la principal fuente de abastecimiento para actividades agrícolas de riego, así como en la creciente demanda para uso doméstico. Para el acuífero correspondiente (localizado en la porción suroriental del Estado de Hidalgo, en el límite con el Estado de Tlaxcala, entre los paralelos $19^{\circ} 35^{\prime}$ y $19^{\circ} 50^{\prime}$ latitud norte y los meridianos $98^{\circ} 11^{\prime}$ y $98^{\circ} 40^{\prime}$ longitud oeste, en una superficie de $733 \mathrm{~km}^{2}$ ), dentro de las soluciones para preservar la disponibilidad hídrica se encuentran la regulación del uso del agua a través de mecanismos de planificación, normativas y leyes que permitan su protección y su distribución en forma racional [1].

La zona mantiene una actividad agrícola tradicional. Sus aguas residuales se vierten sin previo tratamiento en arroyos. Gran parte de esta agua se infiltra al subsuelo debido a la geología de la región, haciendo vulnerable su acuífero a la contaminación. Estudios previos hechos en pozos de abastecimiento público, reportaron concentraciones de Fe y Mn por encima de los límites permisibles fijados por la norma mexicana correspondiente [2], [3] por lo que el interés original del estudio fue evaluar la distribución espacial de estos contaminantes en el acuífero. Durante el análisis de la información se detectó la presencia de fluoruros en el agua por lo que se incluyeron en el estudio.

Se consideran como contaminantes tanto el hierro, el manganeso y los fluoruros en el agua destinada a consumo humano debido a que el hierro le confiere al agua un sabor desagradable, además de la apariencia no estética de la misma.

En el caso de los niños que han estado expuestos a manganeso; y que a elevadas concentraciones se relaciona con incrementos en los niveles de irritabilidad, agresiones, hiperactividad y comportamientos antisociales. Asimismo, el acumulamiento de manganeso en el cerebro puede inducir una forma atípica de enfermedad de Parkinson. La exposición al flúor en cantidades mayores a las reglamentadas, ocasionan fragilidad en los huesos, así como posibles problemas de fertilidad [4].

Debido a la demanda del agua para uso agrícola y para la industria principalmente cervecera y tomando en cuenta que se vierte agua residual sin tratamiento alguno a los cuerpos de agua, cabe inferir que se tendrá un grado de presión hídrica en el acuífero con algún posible grado de contaminación del agua subterránea.

\section{MARCO TEÓRICO}

Geología e Hidrogeología de la zona de estudio

El subsuelo de la zona de Apan se encuentra constituido por una alternancia de tobas, arenas, arcillas, piroclásticos y corrientes de lava (predominantemente de basaltos y andesitas). Su permeabilidad es variable, pero llegan a encontrarse horizontes permeables que permiten la acumulación de agua en el subsuelo, dando origen a un acuífero. 
El acuífero de Apan, se encuentra a una profundidad de 60-70 metros. Se recarga a partir de la infiltración de agua pluvial que se genera en las elevaciones topográficas, la cual circula de la periferia hacia el centro del valle. Existe una salida de agua subterránea en dirección noroeste a la altura de $\mathrm{Cd}$. Sahagún, a través de un estrechamiento topográfico [5].

Técnicas de determinación de contenidos iónicos y muestreo en campo

Se determinó en campo la temperatura del agua, C.E. y $\mathrm{pH}$. Se realizaron determinaciones de S.D.T. calcio, magnesio, sodio, potasio, cloruros, sulfatos, carbonatos, bicarbonatos, fosfatos, dureza de calcio y dureza total en laboratorio acreditato por la Entidad Mexicana de Certificacion, EMA [5].

La recolección de muestras se realizó en envases de plástico para la determinación de S.D.T., calcio, magnesio, sodio, potasio, cloruros y sulfatos.

Las muestras para determinación de metales se recolectaron en envases plásticos de $250 \mathrm{~mL}$ de capacidad acidificadas con $\mathrm{HNO}_{3}$ a $\mathrm{pH}<2$. Todas las muestras fueron conservadas a $4^{\circ} \mathrm{C}$ hasta su análisis.

Las determinaciones de aluminio, arsénico, boro, bario, cadmio, cobre, hierro, manganeso, fosfatos, mercurio, plomo, sílice y zinc así como fluoruros fueron realizadas en laboratorio certificado por la EMA [5] utilizando espectrometría de masas con plasma acoplado inductivamente (ICP/MS) y FIAAS para los fluoruros.

Vega et al., previamente reportaron concentraciones de hierro en el intervalo de $1.45 \mathrm{a}$ $1.6 \mathrm{mg} / \mathrm{L}$ y que fueron muy similares en los pozos evaluados, cuyo fin es de abastecimiento a la población para uso doméstico. Por otra parte, se encontraron valores de manganeso de 3.718-4,050 mg/L. [2]

\section{PARTE EXPERIMENTAL}

Con la información proporcionada por la Comisión Estatal del Agua y Alcantarillado del Estado de Hidalgo, CEAA, se analizó la piezometría del acuífero de Apan, obteniéndose las direcciones predominantes del movimiento del flujo subterráneo (Figura 1). Con base en dichas direcciones, se realizó el diseño del muestreo de los pozos de abastecimiento público/agrícola.

Para dichos pozos seleccionados se analizaron las concentraciones de Fe y Mn presentes encontrándose que el $\mathrm{Mn}$ no es un elemento que rebase las concentraciones máximas permitidas en la mayoría de los pozos. Sin embargo, tanto el $\mathrm{F}$ como el Fe, aunque no rebasan dicha norma, sí se encuentran presentes en la mayoría de los mismos, por lo que el estudio se enfocó en determinar su distribución en el acuífero. Asimismo, se analizaron las facies hidrogeoquímicas de los pozos seleccionados. 


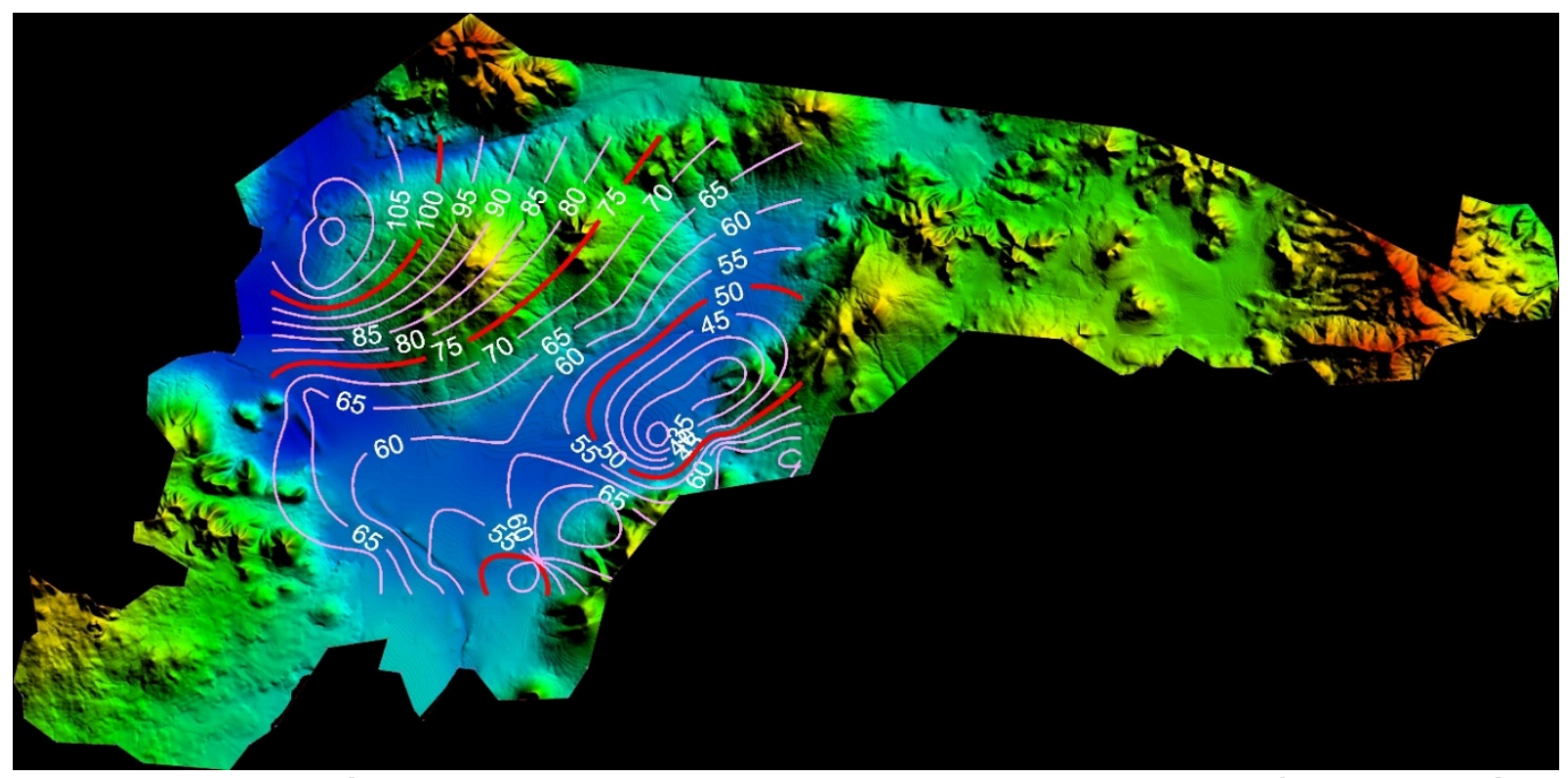

Figura 1. Piezometría del Acuifero de Apan. Adaptado del Estudio geohidrológico del acuífero de Apan, Hidalgo. J.M. Lesser [3].

\section{RESULTADOS}

Para realizar el análisis de los resultados, se tomaron en cuenta los pozos que presentaron las mayores concentraciones de hierro y fluoruros, independientemente del uso industrial, agrícola o doméstico (Tabla 1).

Tabla 1. Resultados del análisis de Fe, Fy Mn.

\begin{tabular}{|c|c|c|c|}
\hline $\begin{array}{c}\text { POZO } \\
\text { No. }\end{array}$ & $\begin{array}{c}\mathrm{Fe} \\
\mathrm{mg} / \mathrm{L}\end{array}$ & $\begin{array}{c}F \\
\mathrm{mg} / \mathrm{L}\end{array}$ & Mn $\mathrm{mg} / \mathrm{L}$ \\
\hline A-2 & 0.022 & 0.027 & ND \\
\hline A-3 & 0.022 & 0.296 & ND \\
\hline$A-5$ & 0.019 & 0.357 & ND \\
\hline$A-7$ & 0.019 & 0.269 & ND \\
\hline$A-8$ & 0.022 & 0.294 & ND \\
\hline A-12 & 0.019 & 0.333 & ND \\
\hline A-13 & 0.023 & 0.371 & ND \\
\hline A-20 & 0.020 & 0.346 & ND \\
\hline A-23 & 0.114 & - & 0.0116 \\
\hline A-24 & 0.040 & - & 0.0034 \\
\hline $\mathrm{A}-30$ & 0.021 & 0.364 & ND \\
\hline A-33 & 0.019 & 0.275 & ND \\
\hline A-34 & 0.018 & 0.244 & ND \\
\hline A-36 & 0.019 & 0.279 & ND \\
\hline A-39 & 0.022 & 0.310 & 0.0164 \\
\hline $\mathrm{A}-40$ & 0.016 & 0.312 & ND \\
\hline
\end{tabular}




\begin{tabular}{|c|l|l|l|}
\hline A-45 & 0.019 & 0.369 & ND \\
\hline A-48 & 0.020 & 0.211 & ND \\
\hline A-50 & 0.017 & 0.438 & ND \\
\hline A-52 & 0.075 & 0.275 & ND \\
\hline A-57 & 0.025 & 0.255 & ND \\
\hline LMP & 0.30 & 1.50 & 0.15 \\
\hline
\end{tabular}

LMP = Límite máximo permisible, $\mathrm{mg} / \mathrm{L}$, para agua de uso y consumo humano. NOM-127-SSA1-1994 (2000) ND= No Detectado

En la figura 2a se muestra la distribución espacial de los fluoruros y en la figura $2 \mathrm{~b}$ la del hierro de acuerdo a las concentraciones extraídas de los pozos seleccionados por la dirección que presenta la piezometría (Figura 1). En ellas se puede observar que el comportamiento de los fluoruros es concéntrico hacia la parte media del acuífero, lo que pudiera indicar algún punto de extracción de agua con volúmenes importantes en la dirección mostrada con lo que habría que analizar más a detalle el tipo de asentamientos que se presentan en la dirección máxima de F.

El comportamiento del Fe, sin embargo, tiende a un comportamiento descendente con respecto a la dirección en la que tiende a moverse el agua (E-O), lo que puede explicarse debido a la geología presente (Figura 2b)

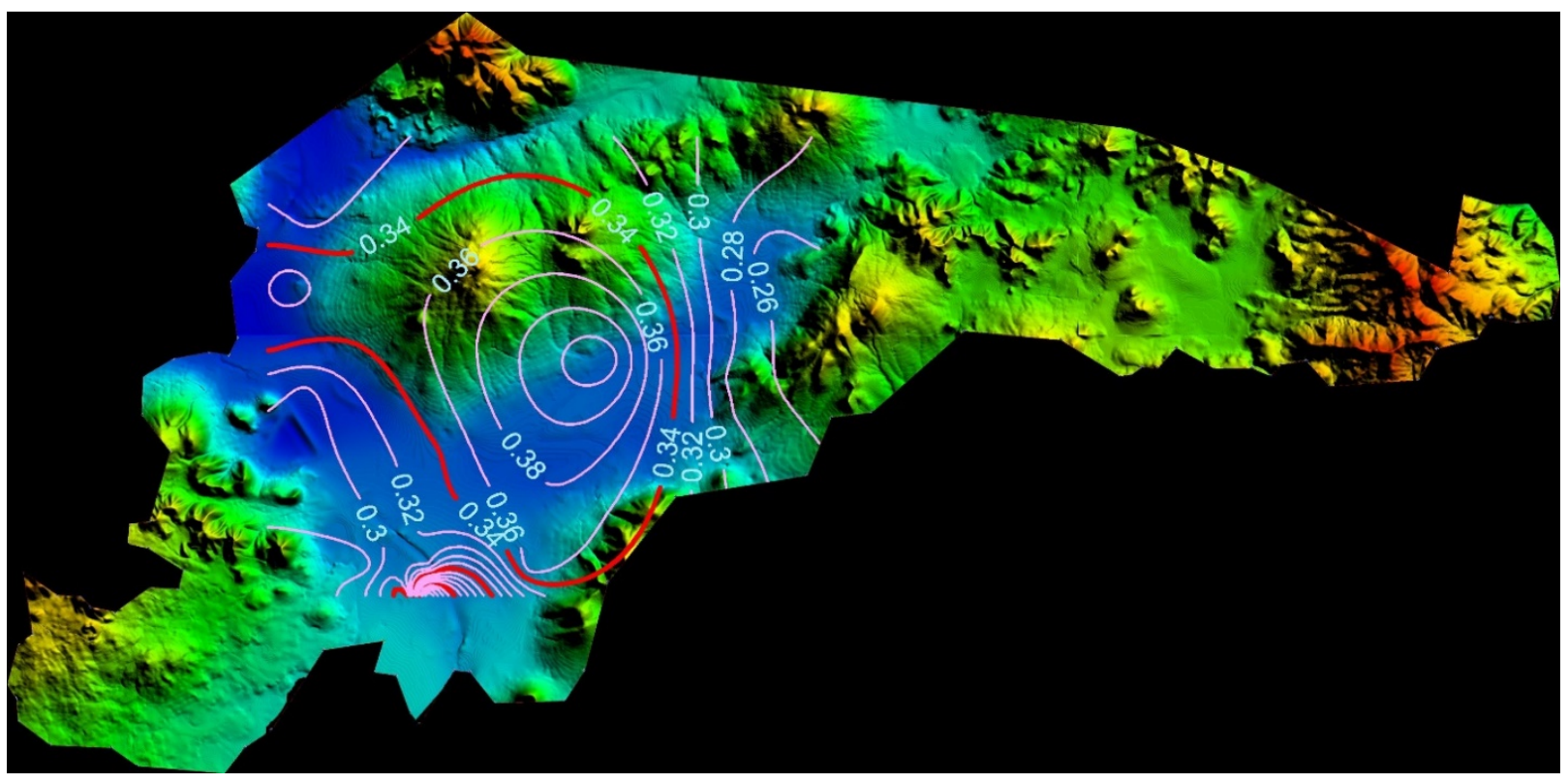

a) Distribución de F 


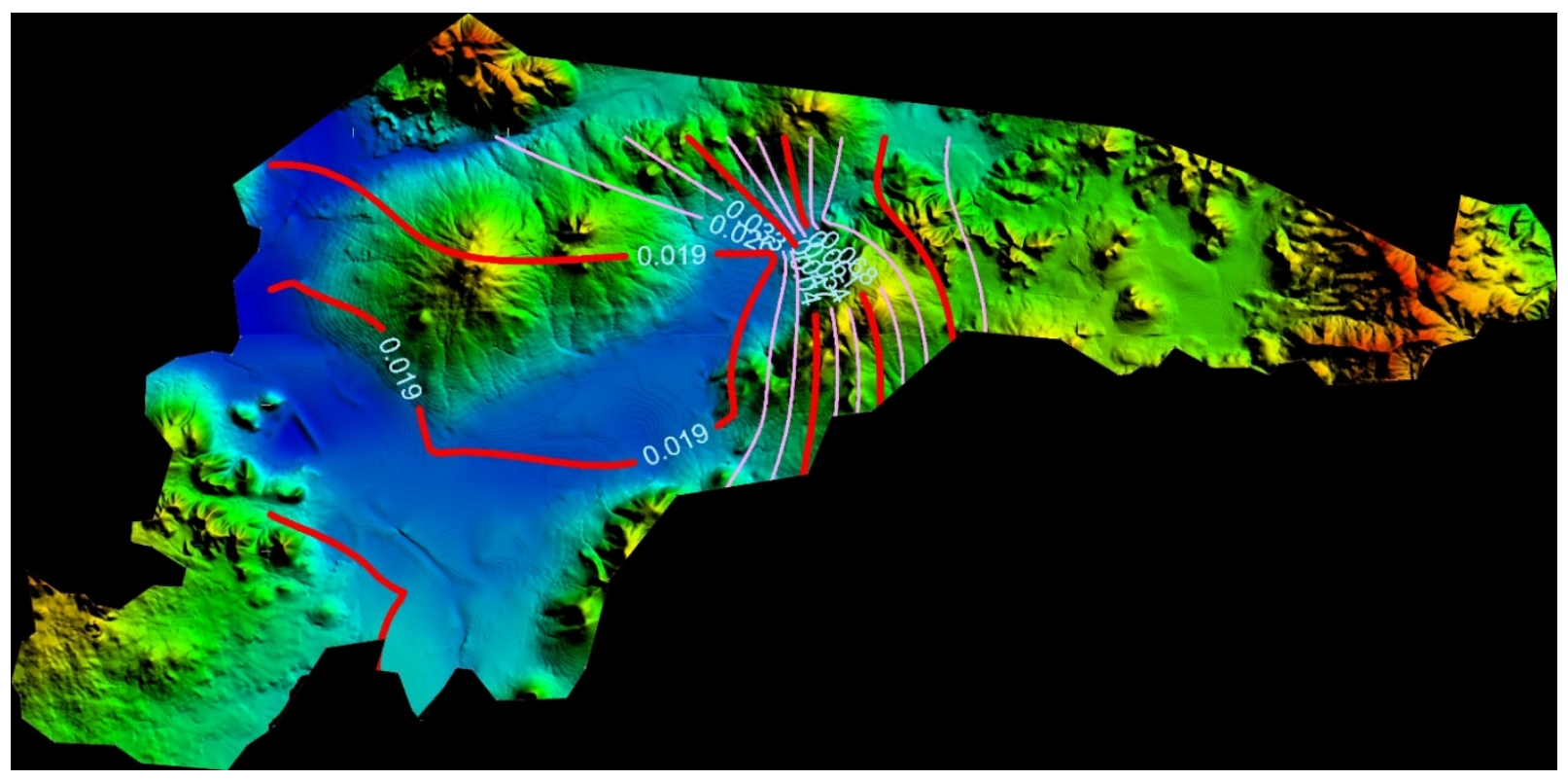

b) Distribución de Fe

Figura 2. Curvas de distribución de hierro y fluoruros en el acuífero de Apan, Hgo.

De acuerdo a la información proporcionada (Figura 3), se corrobora que la facies hidrogeoquímica presente en la dirección del flujo subterráneo es del tipo bicarbonatadamixta $[6,7,8]$ (bicarbonatada cálcica/magnésica a bicarbonatada sódica/cálcica), lo que indica un tipo de agua joven procedente del agua de lluvia y/o de flujo de tipo local con tendencia a un intercambio iónico entre $\mathrm{Ca}$ y $\mathrm{Mg}$ [5], como lo indicó Lesser et al. siendo menor en la zona de recarga aumentando hacia la zona de descarga, lo cual indicaría también una no alteración de las condiciones naturales del acuífero.

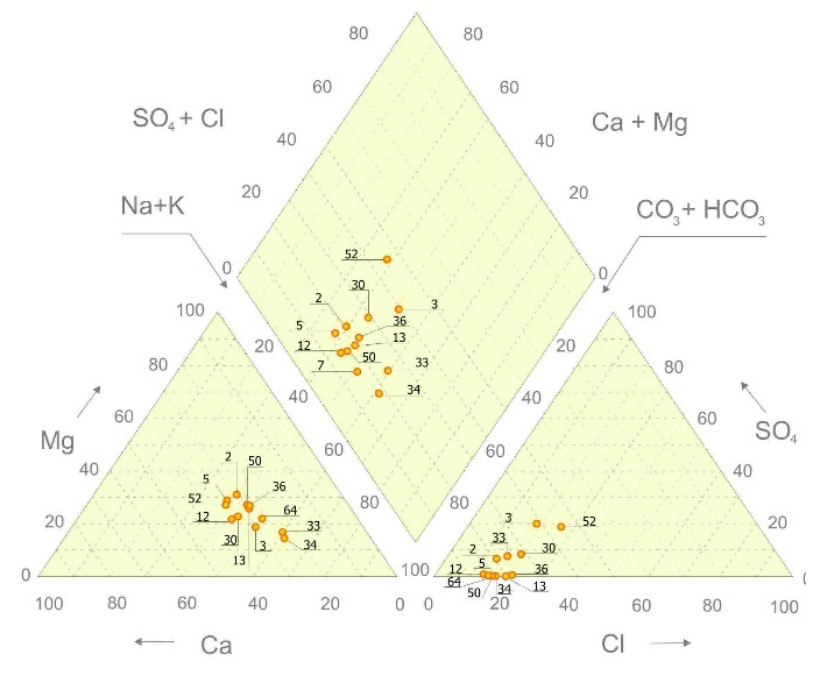

Figura 3. Diagrama de Piper del Acuifero de Apan. Tomado del Estudio geohidrológico del acuífero de Apan, Hidalgo, de J.M. Lesser. Tomo 1. 2007 


\section{CONCLUSIONES}

No se encontró la presencia de niveles por encima de la NOM-127-SSA-1994 para los elementos analizados, sin embargo, dado que la extracción de agua subterránea se ha incrementado en forma considerable debido al asentamiento de industrias de tipo cervecero, se sugiere realizar un seguimiento del comportamiento tanto de la piezometría como de los niveles de metales estudiados.

Respecto a los resultados de hierro y manganeso, el primero de ellos se encontró en concentraciones que van de 0.016 a $0.114 \mathrm{mg} / \mathrm{L}$, mientras que el manganeso, en la mayor parte de las muestras analizadas no fue detectado y solamente en tres de ellas se encontró en concentraciones que van de 0.0034 a $0.0164 \mathrm{mg} / \mathrm{L}$. En ambos casos, las concentraciones se encuentran por debajo del límite máximo permitido de acuerdo a la NOM-127-SSA1-1994 (2000).

Los fluoruros se encontraron en concentraciones que van de 0.027 a $0.438 \mathrm{mg} / \mathrm{L}$, valores que se encuentran por debajo del límite máximo permitido para el agua de uso y consumo humano.

El Fe presenta una orientación espacial en dirección contraria a la circulación del agua $\mathrm{E}-\mathrm{O}$. Los fluoruros presentan una tendencia ascendente con dirección concéntrica.

El acuífero presenta una calidad adecuada para uso y consumo humano en su mayor parte.

\section{AGRADECIMIENTOS}

Los autores agradecen al fondo PRODEP por los recursos otorgados para la realización del estudio, así como al Ing. Santiago Arellano y al personal de la Comisión Estatal del Agua y Alcantarillado del Estado de Hidalgo por su disponibilidad para facilitar la información requerida.

\section{BIBLIOGRAFÍA}

[1]. CONAGUA. Actualización de la disponibilidad media anual de agua en el acuífero Apan (1320), Estado de Hidalgo. Comisión Nacional del Agua, México D. F.

Último acceso: junio 2019, https://www.gob.mx/cms/uploads/attachment/file/103069/DR_1320.pdf

[2]. A. G. Vega, G. Zárate, P. Yáñez, S. H. González. Determinación de hierro y manganeso en el agua subterránea del municipio de Apan, Hidalgo,México. Reaxion. 5(1) (2017). Último acceso: junio 2019. http://reaxion.utleon.edu.mx/Art_Determinacion_de_hierro_y_manganeso_en_el_agua_subt erranea_del_municipio_de_Apan_Hidalgo_Mexico.html

[3]. Norma Oficial Mexicana Nom-127-SSA1-1994, "Salud Ambiental, Agua Para Uso Y Consumo Humano-Límites Permisibles De Calidad Y Tratamientos A Que Debe Someterse El Agua Para Su Potabilización". (2000) 3-4

[4]. Departamento de Salud y Servicios Humanos de los EE.UU. Agencia para Sustancias Tóxicas y el Registro de Enfermedades División de Toxicología, Atlanta (2000) 1-2. Último 
acceso: junio 2019. https://www.atsdr.cdc.gov/es/toxfaqs/es_tfacts151.html. https://www.atsdr.cdc.gov/es/toxfaqs/es_tfacts11.html

[5]. J.M. Lesser. Estudio geohidrológico del acuífero de Apan, Hidalgo. Tomo 1 (2007) 95-103. Archivo interno de la Comisión Estatal del Agua y Alcantarillado del Estado de Hidalgo.

[6]. R. Huízar, T. Méndez, R. Madrid. Revista Mexicana de Ciencias Geológicas. 16(1) (1999) 8996.

[7]. A. Enríquez, F. Sánchez, R. H. Alvarez, L. M. Salazar, D. A. Vilchis, T. García. Revista Mexicana de Ciencias Geológicas. Evaluación hidrogeológica de la subcuenca de Tecocomulco, Estados de Hidalgo, Puebla y Tlaxcala, México. 18(1) (2001), 55-73.

[8]. P. D. Roy, M. Caballero, R. Lozano, W. Smykatz-Kloss. Geochemistry of late quaternary sediments from Tecocomulco lake, central Mexico: Implication to chemical weathering and provenance. Chemie der erde-geochemistry, 68(4) (2008). 383-393. 